\title{
2 DOF Resolution Adjustment Laser Position Sensor
}

\author{
A. Shaik ${ }^{1}$, N. S. Tlale ${ }^{1}$, G. Bright ${ }^{2}$ \\ ${ }^{1}$ Department of Material Science and Manufacturing \\ Council for Scientific and Industrial Research \\ Pretoria, 0001, South Africa \\ ${ }^{2}$ Department of Mechanical Engineering \\ University of KwaZulu-Natal \\ Durban, 4001, South Africa
}

\begin{abstract}
A low cost sensor system is envisaged that has 2 distinct routes for resolution enhancement in position detection. The main area for application of the sensor design would be in the food processing and packaging industry where position resolution of the end effector is in the millimeter to submillimeter range. The sensor concept consists of a grid of laser light detectors attached to the stationary base and a grid of lasers attached to the mobile end effector. The photo detectors on the base provide one level for position detection enhancement, i.e. the density of the sensor array. The second degree of freedom in improvement comes from the strategic positioning of a set of lasers attached to the end effector.
\end{abstract}

\section{INTRODUCTION}

Many existing automated multi-axis machines operate under overall 'open loop' control. The exact position in space of the end effecter or tool head is not sensed directly. With knowledge of the robot's initial position, the control system uses differential measurements from sensors on axes that track linear translation and rotations of shafts/gears, to track the position of the end effector. It then uses this information to plot a trajectory to future positions. A direct end effector sensing system would provide additional data for positioning. Errors in the control system brought on by errors in the actuator sensors may then be corrected, to achieve better positioning accuracy and repeatability. This however, is dependent on the resolution of the sensor system involved and the positioning capability of the robot. [1]

The design of the robot and sensor system were specific to the agricultural and food processing/packaging industry. For this application robot speed is paramount, accuracy and repeatability are less stringent but within lie within certain tolerances, roughly in the millimeter range. The sensor system for such an application needs to be robust for the environment in which it is intended to work. This environment is subject to conditions of humidity, vibration and contaminants. An additional sensor system providing direct end effector position location adds sensor redundancy to the control system, thereby improving the ability of accurate positioning.

\section{AN OVERVIEW OF CURRENT LOCATION SENSING METHODS AND TECHNOLOGIES}

There are numerous technologies available that can be used to locate objects in space. They are distinguished from one another by the use of different media, transducers and processing techniques. The common problems are resolution and the type of media used. Resolution is always an issue with measurement and in this particular case refers to the maximum positional error possible with the technology being discussed. With regard to the medium or transducer stimulant, some media may prove undesirable; for instance using an ultrasound triangulation system in a small closed environment.

Triangulation - This mathematical technique is used in most technologies to determine position of objects, and uses geometry to calculate position by considering the properties of triangles to compute locations. There are 2 subcategories of triangulation i.e. lateration and angulation.

The term lateration is used for distance measurements. It computes the position of an object by measuring its distance from multiple reference positions. Calculating an object's position in $2 \mathrm{D}$ requires distance measurements from 3 noncollinear points. For 3D measurements, distances from 4 noncoplanar points are required. There are three general approaches to measuring the distances required by the lateration technique.

Direct - Direct measurement of distance. Direct distance measurements are simple to understand but may prove to be difficult or even impossible to obtain if the distances are large.

Time of Flight (TOF) - Measuring distance from an object to some point $\mathrm{P}$ using TOF means measuring the time it takes a signal to travel between the object and a point $\mathrm{P}$ at a known velocity. The problem is made more complex if the object itself is moving. It is possible to make the calculations if there is a known mathematical function for the acceleration. If however it varies arbitrarily then it is impossible to find a solution. Other factors have to be considered to get an accurate answer, for instance if the signal used is ultrasonic, such as air medium density, temperature and humidity. 
Reflection is another problem as direct and reflected signals look identical. Bats and other creatures that use sonic vision statistically filter reflected measurements by averaging multiple receivers' measurements and observing the environment's reflective properties. Another issue in taking TOF measurements is the time reference. When only one measurement is needed, as with round-trip or radar reflections, timing is simple because the transmitting object is also the receiver and must maintain its own time with sufficient precision to compute the distance. In other systems (like GPS) where the receiver and transmitter are on different objects they must be synchronized precisely to get an accurate time measurement of distance.

Attenuation - The intensity of an emitted signal decreases as the distance from the emission source increases. The decrease relative to the original intensity is the attenuation. Given a function correlating attenuation and distance for a type of emission as well as the original strength of the emission, it is possible to estimate the distance from an object to some point $\mathrm{P}$ by measuring the strength of the emission when it reaches $\mathrm{P}$. Signal propagation issues such as reflection, refraction, and multi-path cause the attenuation to correlate poorly with distance resulting in inaccurate and imprecise distance estimates. [2, 3, 4]

The term angulation is used for angular measurements, and these are used for determining the position of an object. In general, 2D angulation requires two angle measurements and one length measurement such as the distance between the reference points. In 3D, one length measurement, one azimuth measurement, and two angle measurements are needed to specify a position. Angulation implementations sometimes choose to designate a constant reference vector (e.g. magnetic north) as $0^{\circ}$. Phased antenna arrays are an excellent enabling technology for the angulation technique. Multiple antennas with known separation measure the time of arrival of a signal. Given the differences in arrival times and the geometry of the receiving array, it is then possible to compute the angle from which the emission originated. If there are enough elements in the array and large enough separations, the angulation calculation can be performed. [2, 3, 4]

GPS - The operation of the GPS system is based on trilateration. The accuracy of a position determined with GPS depends on the type of receiver. Most hand-held GPS units have an accuracy of about 10-20 m (spherical radius).

DGPS uses two GPS receivers. Observations made by a known stationary location (base or reference) is used to correct the data received by a GPS unit at an unknown location (rover). DGPS generally achieves an accuracy of less than 1 meter. [8] Presents a cheap solution with errors of less than $5 \mathrm{~cm}$.

There are also other flavours of GPS such as Assisted GPS (AGPS) that utilizes an assistance server to aid in position determination, Indoor GPS or high sensitivity GPS, which is a combination of AGPS and massive parallel correlation (to solve the indoor multi-path problem) and Laser indoor GPS systems. [5, 6, 7, 9]

Networks - Bluetooth, WiFi and Cellular networks provide a means for location sensing. Bluetooth devices form mini-cells, and with a sufficient number of Bluetooth cells (access points) installed, the position of a transmitter can be deduced by knowing the cell with which a device is communicating (or location base stations in a WiFi network), discussed in [10] for locating people in buildings. [11] Also takes into account signal strength from the access points. Since the transmitted signal energy decreases almost proportionally with the distance between stations and mobile terminals, this relation can be used to determine the distance from a particular node. These Bluetooth networks have an accuracy ranging from 2 to $5 \mathrm{~m}$ and is subject to the number of cells installed in the region and the spacing between them. $[12,13]$

Interferometry - This is the applied science of combining two or more waves, which are said to interfere with each other. The interference pattern is considered a state with amplitude and phase which depends on the amplitude and phase of all the contributing waves. The interferometer can measure displacements to a resolution within a fraction of the wavelength of light. It has enabled Micro- and Nano-scale measurements of position or movement. [14]

Grid Encoders - An optical grid encoder is capable of 2D dynamic measurements with high accuracy and excellent repeatability. The grid encoder is composed of a grid plate with a waffle-type grating of closely spaced lines ( $4 \mu \mathrm{m}$ signal period) and a non-contact scanning head which is able to measure translations in two directions. This system measures the relative planar motion of the two bodies for any curvilinear path in the plane of the mounting base with a resolution of 4 $\mathrm{nm}$ and to within an accuracy of $\pm 2 \mu \mathrm{m}$. [15]

Image Processing - Imaging methods use cameras and sophisticated software to determine position location. [16] Discusses a low cost solution employing a camera and LCD screen to locate an object's coordinates in 2D with high accuracy. The LCD screen displays an image, a small circle or cross somewhere on its surface. The camera which is attached to the end effector then tries to align this shape in some way with the image it produces. It can also be used to determine $2 \mathrm{D}$ orientation, using pattern projections.

The scene analysis location sensing technique uses features of a scene observed from a particular vantage point to draw conclusions about the location of the observer or of objects in the scene. Usually the observed scenes are simplified to obtain features that are easy to represent and compare. The advantage of scene analysis is that the location of objects can be inferred using passive observation and features that do not correspond to geometric angles or distances. The disadvantage of scene analysis is that the observer needs to have access to the features of the environment against which it will compare its observed scenes. Furthermore, changes to the environment in 
a way that alters the perceived features of the scenes may necessitate reconstruction of the predefined dataset or retrieval of an entirely new dataset. $[17,18]$

SLAM - Simultaneous Localization and Mapping (SLAM) is a technique used by robots and autonomous vehicles to build up a map within an unknown environment while at the same time keeping track of its current position from various sensors. If at the next iteration of map building the measured distance and direction traveled has a slight inaccuracy, then any features being added to the map will contain corresponding errors. Some of the statistical techniques used in SLAM include Kalman filters, particle filters (a.k.a. Monte Carlo methods) and scan matching of range data. SLAM usually uses laser range finders or sonar sensors to build the map. However VSLAM (visual simultaneous localization and mapping) uses entirely visual means. $[19,20]$

Other - Displacement measuring instruments utilizing eddy currents, capacitive and inductive properties exist, but are not as widely spread as the technologies mentioned.

Accelerometers and Gyroscopes are used to determine position and orientation. They are capable of measuring the change in acceleration of a body. An integration of this yields velocity and a second integration yields distance traveled. Keeping track of these parameters and having been given an initial position for the object, its current position can be inferred. This system is usually used in conjunction with other techniques/technologies such as GPS and radar.

\section{SENSOR SySTEM CONCEPT}

Passive systems, or imaging methods require lots of processing power, complex software and expensive cameras. Furthermore, with current technology the systems are not real time. An active sensor system was therefore designed, that is one that transmits a signal and then receives it with a sensor array at another location, and then computes position.

The problem of locating the end effector of a robot in real world space was first reduced to finding its position in a 2D plane with regard to a point reference. Once accomplished the general problem is solved by attaching two $2 \mathrm{D}$ planes at right angles. With such an arrangement 2 axes coincide and if the reference point of each plane coincides, the result is a 3 axis sensor system for position location in $3 \mathrm{D}$ space.

After consideration of the available physical quantities used when locating objects, a laser light stimulant was chosen for the task at hand. A laser light sensor can be conditioned to provide a digital output. Most sensors used for tracking are analogue in nature and require digitization for use in digital systems. The varying value indicating light intensity is irrelevant, as all that is required is a value saying that that sensor has been stimulated by laser light. Furthermore analogue signals are compromised by atmospheric effects, temperature, humidity and unshielded noise from surrounding machinery. Triangulation utilizing radio, ultrasound or infrared waves is not suitable as multiple reflections from surrounding surfaces produce incorrect readings.

The defining component of this sensor system is a grid of laser light detectors. The detectors need to have a narrow sensitivity wavelength bandwidth as well as viewing angle to prevent wrongful stimulation and spurious results. The designed sensor concept utilizes a direct approach, with a laser or set of lasers, attached to the end effector and the sensor grid (the sensor plane with sensors spaced equally in rows and columns) mounted directly above it, to the side or directly below. This was a natural choice as the coherent nature of laser light aids the task of finding the end effector in 2D if the laser beam remains perpendicular to the sensor plane at all times. The end effector's location is the same as the sensor which is stimulated. This sensor system requires only bit ( 1 or 0$)$ information for each sensor. Each sensor is either stimulated (switched on) or not stimulated (switched off), and a stimulated sensor indicates position on the plane. This makes data processing and transfer far simpler and makes control easier. The resolution is limited to the spacing between sensors. If the spot light is smaller than the spacing between sensors, there will be a dead zone between sensors where beam tracking will be lost completely. The laser light detectors are phototransistors. Current fabrication techniques can accommodate hundreds of millions of transistors on a sliver of silicon but at high cost. Fabrication methods can be used to construct a detector screen with an exceptional and practical resolution. The current accuracy of the IRB 340 Flex Picker, a rapid pick and place machine, is $0.1 \mathrm{~mm}$. The lower limit on screen resolution for absolute $2 \mathrm{D}$ positioning would then be 400 phototransistors per square $\mathrm{mm}$, a screen with twice the resolution of the positioning accuracy of the robot. A hybrid type system (combining this end effector sensor with conventional motor encoders and software position fixing) would involve a sensor grid with a comparatively smaller resolution. Each sensor provides a checkpoint. Knowing the exact spatial distance between these detectors provides the controller with a means to limit the errors incurred. Instead of accumulating errors from one extremity to the next, errors only exist between successive detectors.

The array of data has to be placed in a data format or byte structure to facilitate processing. This is made possible by parallel to serial data converters. As a numerical example consider first the workspace of the IRB 340 Flex Picker from ABB Automation. The specified workspace envelope is a cylinder with diameter $1130 \mathrm{~mm}$ and height of $250 \mathrm{~mm}$. A $1150 \mathrm{~mm} \times 1150 \mathrm{~mm}$ screen would be large enough to track the end effector in its specified workspace. Using the same resolution as before i.e. 400 detectors per $\mathrm{mm}$ squared, there would be 529000000 detectors on the screen. This sensor grid consists if 23000 rows and 23000 columns. A 4 byte data format ( 2 bytes for the row and 2 bytes for the column) would be more than sufficient to indicate any single stimulated sensor to an external control system.

Figure 1 displays high level architecture of the detector screen. 


\section{SENSOR SYSTEM RESOLUTION}

The sensor system designed that was designed and built has a resolution of $2.5 \mathrm{~mm}$. The detector screens have a resolution of $10 \mathrm{~mm}$. The improvement in resolution is due to the use of a laser module, one laser module per detector screen, at the end effector. Each laser module has 12 lasers with a particular arrangement to provide the sensor system with the resolution stated. To explain how this is achieved an animated depiction of the laser projection onto the detector screen is illustrated in Figures 2, 3 and 4.

The blue circles represent the sensors. The red and green circles represent the lasers. The central laser with the blue bull's eye, henceforth know as BE, is the tracking point or end effector reference. The relative spacing of these lasers are shown in Figure 2. The 4 inner lasers lie on grid points with a grid spacing of $15 \mathrm{~mm}$. These lasers improve the resolution of the detector system to $5 \mathrm{~mm}$, and this laser grid will be known as G5. These lasers are represented by the BE and 3 green circles in Figure $2 \mathrm{~b}$. The outer lasers lie on grid points with a grid spacing of $22.5 \mathrm{~mm}$ and improve the resolution of the sensor system to $2.5 \mathrm{~mm}$, and this grid will be known as G2.5. Grid G2.5 has to be used in conjunction with grid G5 and is represented by BE and the red circles in Figure 2 b.

The black solid lines of Figure $2 \mathrm{~b}$ are reference lines. The dashed lines represent the $2.5 \mathrm{~mm}$ resolution. Figure 2 a shows the mechanical component used to mount the lasers.

Suppose initially BE is at sensor $s(i, j)$, in Figure $2 \mathrm{~b}$. If it moves $5 \mathrm{~mm}$ to the right it no longer lies on $s(i, j)$, however the green circle on the same y grid line lies on sensor $s(i+2, j)$ (Figure $3 \mathrm{a}$ ). If BE were to move $5 \mathrm{~mm}$ to the left, that same green circle would lie on sensor $s(i+1, j)$ (Figure $3 \mathrm{~b}$ ). So any horizontal $5 \mathrm{~mm}$ displacement can be tracked; this is the maximum distance the end effector would have to move horizontally before another sensor indicates position.

Similarly $5 \mathrm{~mm}$ vertical displacements can be tracked (shown in Figure $3 \mathrm{c}, \mathrm{d}$ ). Combined displacements can also be tracked. Suppose BE moves $5 \mathrm{~mm}$ to the right and $5 \mathrm{~mm}$ up, then the bottom left hand green circle lies on sensor $s(i+2, j-1)$ (Figure $3 \mathrm{e})$. If $\mathrm{BE}$ is moved $5 \mathrm{~mm}$ to the left and $5 \mathrm{~mm}$ down, then that same green circle lies on sensor $s(i+1, j-2)$ (Figure $3 \mathrm{f}$ ).

\section{Sensor Screen Architecture}

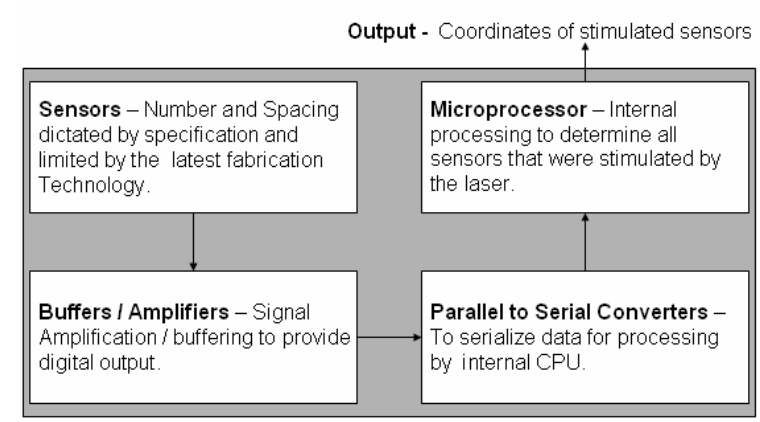

Figure 1. High level architecture of detector screen

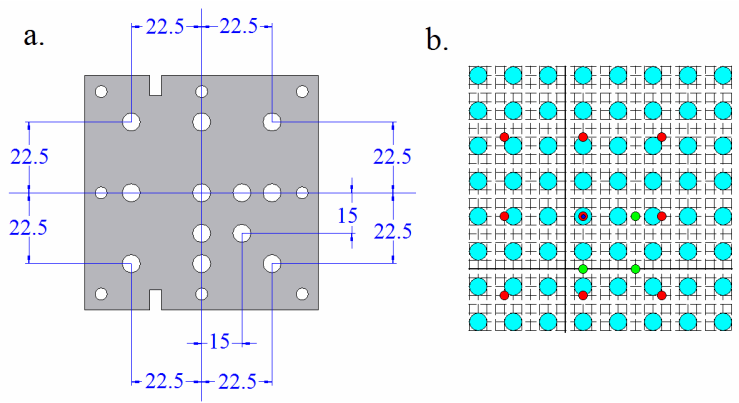

Figure 2. Laser grid

a. Laser module mounting indicating dimensions and laser positions b. Laser grid projection onto detector plane, centred on $s(i, j)$

The $2.5 \mathrm{~mm}$ resolution is proved as follows. Start of at position in Figure $2 \mathrm{~b}$. Move $2.5 \mathrm{~mm}$ to the right. The laser sharing the same y grid line to the far left of BE then lies on sensor $s(i-2, j)$ (Figure 4 a). If BE is moved $2.5 \mathrm{~mm}$ down then the laser at the top left hand corner lies on sensor $s(i-2, j+2)$ (Figure $4 \mathrm{~b}$ ). If BE is now moved $2.5 \mathrm{~mm}$ to the left the laser sharing the same $\mathrm{x}$ grid line directly above $\mathrm{BE}$ moves onto sensor $s(i, j+2)$ (Figure $4 \mathrm{c}$ ). The pattern can now be seen clearly. As BE moves about the $2.5 \mathrm{~mm}$ grid surrounding sensor $s(i, j)$ the lasers on $\mathrm{G} 2.5$ hit sensors lying on the $20 \mathrm{~mm}$ grid surrounding sensor $s(i, j)$ (i.e. $s(i \pm m, j \pm n)$ where $\mathrm{m}, \mathrm{n}=0$ or 2 as shown in Figure 4 a to g). So any $2.5 \mathrm{~mm}$ vertical, horizontal or combined displacement can be sensed. Similarly displacements of $7.5 \mathrm{~mm}$ can be tracked.

The meaning of stimulated sensors does not refer to the exact position of the reference point $\mathrm{BE}$ but rather displacements from that point. For PKM machine there are sensors that monitor the angular positions of the upper legs. Those measurements together with these end effector displacement error readings provide improved end effector tracking.

\section{ADVANTAGES AND DisAdVANTAGES OF SENSOR CONCEPT}

Advantages:

- Detector screen of any practical size can be built from modular components.

- Resolution is independent of screen size (due to modularity), and the detector screens can be scaled up maintaining the exact resolution designed.

- Data is purely digital as the sensors are either on (1) or off (0).

- It facilitates 2D or 3D tracking.

- Laser module on end effector provides a 2 fold increase in resolution of the sensor screen. 
a.

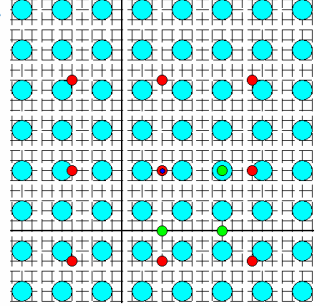

c.

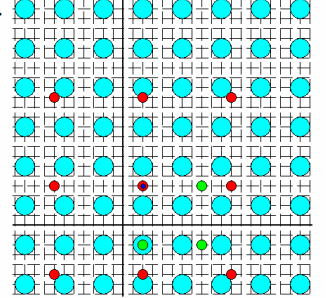

e.

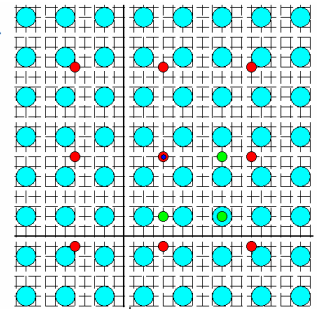

b.

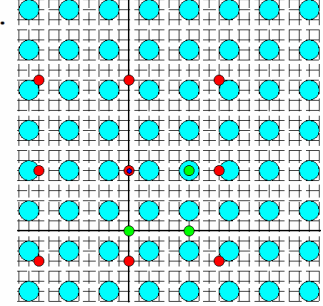

d.

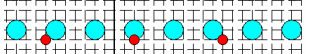

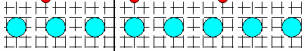

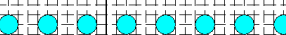

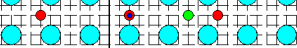

OHO+O

Q

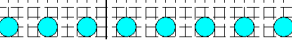

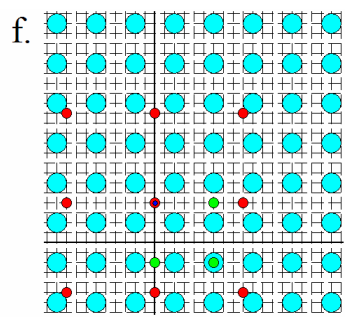

Figure 3. Depiction of laser grid projection onto detector plane for $5 \mathrm{~mm}$ resolution

a. BE moved $5 \mathrm{~mm}$ to the right of $s(i, j), s(i+2, j)$ detects laser

b. BE moved $5 \mathrm{~mm}$ to the left of $s(i, j), s(i+1, j)$ detects laser

c. BE moved $5 \mathrm{~mm}$ down from $s(i, j), s(i, j-2)$ detects laser

d. BE moved $5 \mathrm{~mm}$ up from $s(i, j), s(i, j-1)$ detects laser

e. BE moved $5 \mathrm{~mm}$ up $\&$ to the right of $s(i, j), s(i+2, j-1)$ detects laser

f. BE moved $5 \mathrm{~mm}$ down \& to the left of $s(i, j), s(i+1, j-2)$ detects laser

a.

.

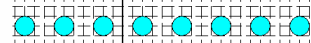

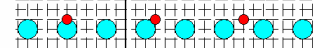

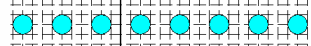

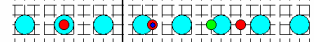
OHOI

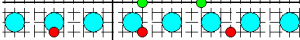

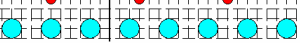

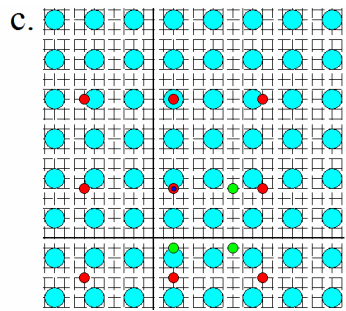

b. 4 atol 4 q

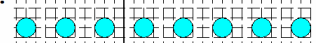

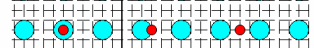
- 4 + H.1.

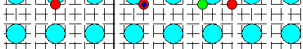

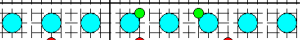

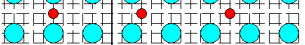

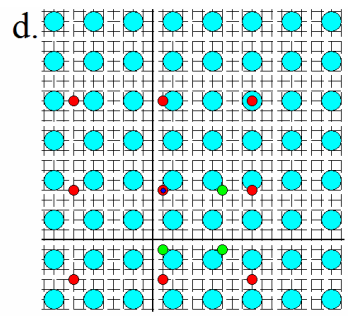

e.

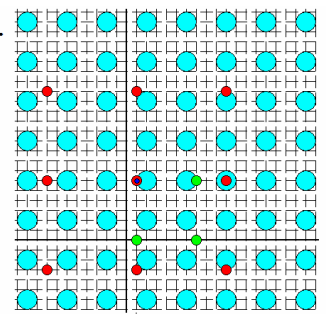

f. 4 Q

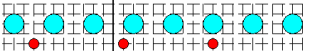
H.
HOA

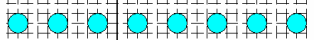

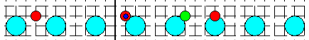

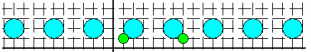
tod

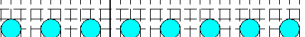
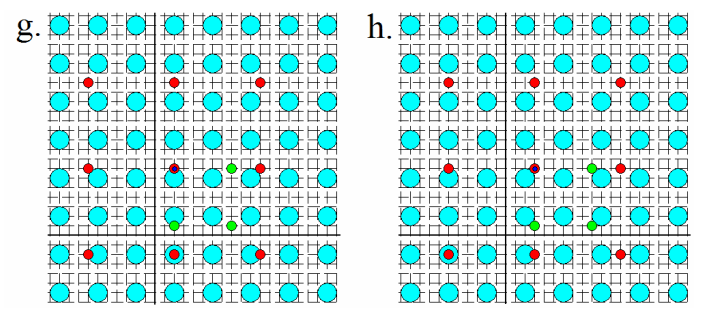

Figure 4. Depiction of laser grid projection onto detector plane for $2.5 \mathrm{~mm}$ resolution

a. BE moved $2.5 \mathrm{~mm}$ to the right of $s(i, j), s(i-2, j)$ detects laser

b. BE then moved $2.5 \mathrm{~mm}$ down, $s(i-2, j+2)$ detects laser

c. BE moved $2.5 \mathrm{~mm}$ to the left, $s(i, j+2)$ detects laser

d. BE moved $2.5 \mathrm{~mm}$ to the left, $s(i, j), s(i+2, j+2)$ detects laser

e. BE moved $2.5 \mathrm{~mm}$ up, $s(i+2, j)$ detects laser

f. BE moved $2.5 \mathrm{~mm}$ up, $s(i+2, j-2)$ detects laser

g. BE moved $2.5 \mathrm{~mm}$ to the right, $s(i, j-2)$ detects laser

h. BE moved $2.5 \mathrm{~mm}$ to the right, $s(i-2, j-2)$ detects laser

Disadvantages:

- For 3D tracking 2 screens are needed, and the system becomes twice as expensive.

- The lasers must remain perpendicular to the detector screens.

- This sensor must fit the space and mounting constraints imposed by the manufacturing system.

\section{PROTOTYPE}

A scale model PKM was constructed (that accomplishes translational positioning only) and 2 scalable modular detector screens mounted at right angles to each other. Figure 5 displays the test rig, and the sensor design which is currently being evaluated.

\section{CONCLUSION}

This paper discussed current technologies used for position location. Most of the equipment used for each technology was unsuitable for the task of locating a robotic end effector for one of more of the following reasons: the systems were too expensive, slow, inaccurate or bulky. From this investigation the idea of active laser image projection was conceptualized. The sensor design was then discussed and its resolution capability proved. The concept has 2 possible routes to enhance resolution, either by increasing the density of sensors on the sensor screens or by increasing the number of lasers on the end effector and its strategic positioning. Thus far the laser module has increased the sensor resolution by a factor of 4 
and its effect is exponential. However the number of lasers in each surrounding layer also increases exponentially. The concept does have potential for application in certain industries, such as the food packaging industry where robust position location determination of the end effector is required, and position resolution is in the millimeter range.

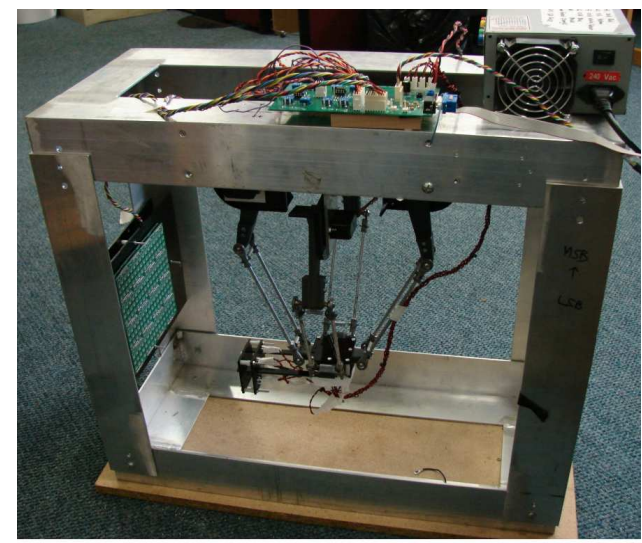

a.

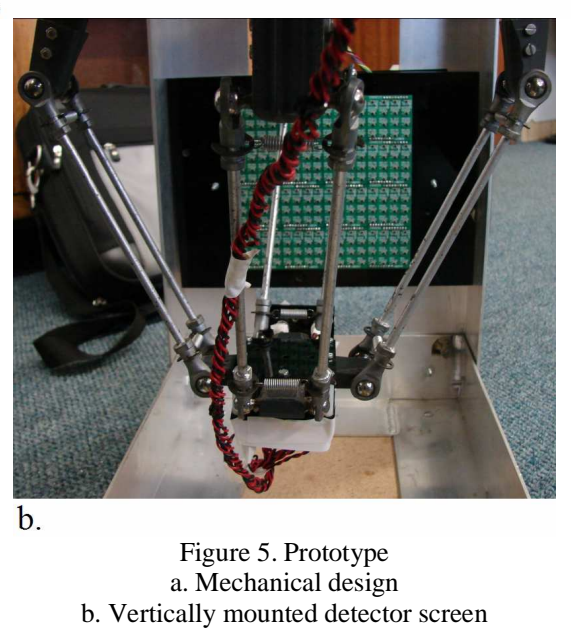

\section{REFERENCES}

[1] "Parallel Robot Design Incorporating A Direct End Effector Sensing System", Ahmed Shaik and Nkgatho S. Tlale, Council for Scientific and Industrial Research, CSIR 0001, Pretoria, South Africa; Glen Bright, School of Mechanical Engineering, UKZN-4001, Durban, South Africa; International Journal of Intelligent Systems Technologies and Applications, Vol. 2, Nos. 3/4, 2008, pp. $368-385$.

[2] "Location Sensing Techniques", by Jeffrey Hightower and Gaetano Borriello, Computer Science and Engineering, University of Washington. Technical report, UNCSE 01-07-01, July 2001.

[3] "On the Potential Use of Mobile Positioning Technologies in Indoor Environments", by A. Pateli, G. M. Giaglis, K. Fouskas, P. Kourouthanassis and A. Tsamakos; Proceedings of 15th Bled Electronic Commerce Conference -e-Reality: Constructing the e-Economy; Bled, Slovenia, April 2002.
[4] "A Taxonomy of Indoor and Outdoor Positioning Techniques for Mobile Location Services", by G.M. Giaglis, V. Zeimpekis, and G. Lekakos. ACM SIGECOM Exchanges, Vol. 3, No. 4, p19-27, August 2003.

[5] "The GPS Manual: Principles \& Applications", by S. Dye and F. Baylin; first edition 1997. Baylin-Gale Productions.

[6] "Understanding the GPS: An Introduction to the Global Positioning System”, by G. T. French; first edition, 1997.

[7] "Location Sensing Technologies and Applications", by George Roussos. School of Computer Science and Information Systems, Birkbeck College, University of London, November 2002.

[8] "High Precision GPS Guidance of Mobile Robots" by R. Willgoss, V. Rosenfeld and J. Billingsley. ACRA - Australasian Conference on Robotics and Automation, August 2003.

[9] "Indoor GPS Technology" by Frank van Diggelen and Charles Abraham, Global Locate, Inc. Presented at CTIA Wireless-Agenda, Dallas, USA, May 2001.

[10] "Indoor Positioning Systems in Healthcare, a Basic Overview of Technologies" by M. Dempsey; Radianse Inc. CIMIT - Innovative Technology for Medicine, June 2003.

[11] "In-building location using Bluetooth" by Miguel Rodriguez, Juan P. Pece, Carlos J. Escudero. Departamento de Electronica e Sistemas, Universidade da Coruna, Spain. INWAN - International Workshop on Wireless Ad-Hoc Networks, London, UK, May 2005.

[12] "Indoor and Outdoor Positioning in Mobile Environments - A Review and some Investigations on Wlan-Positioning" by R. Bill, C. Cap, M. Kofahl and T. Mundt. University Rostock, Germany. International Workshop on Ubiquitous Geographical Information Sciences, Vol. 10, No. 2, p91 -98, Gavle, Sweden, June 2004.

[13] "Location Awareness in Ad Hoc Wireless Mobile Networks", by Y. Tseng, S. Wu, W. Liao and C. Chao. IEEE Computer, Vol. 34, No. 6, p.46-52, June 2001.

[14] "Displacement Measuring Interferometers Provide Precise Metrology", by D. Musinski. Laser Focus World, Vol. 39, No. 12, December 2003.

[15] "A new sensor for the micro-metre-level measurement of threedimensional, dynamic contours," by Tony Schmitz and John Ziegert; Machine Tool Research Center, Department of Mechanical Engineering, University of Florida, Gainesville, USA. Measurement Science and Technology, IOP - Institute of Physics - Electronic Journals, Vol. 10, p51-62, Feb. 1999.

[16] “Active Vision/Display Sensors for Precision Positioning” by J. Ziegert. University of Florida, USA. A project proposal to the NSF - National Science Foundation, September 2005.

[17] "Image Classifiers for Scene Analysis", by B. L. Saux and G. Amato. Computational Imaging and Vision, Vol. 32, p39-44, March 2006.

[18] "Visual Schemas in Object Recognition and Scene Analysis", by R Miikkulainen and W. K. Leow. The handbook of brain theory and neural networks, p1029-1031, July 1998

[19] "Simultaneous Map Building and Localization for an Autonomous Mobile Robot", by J. J. Leonard and H. F. Durrant-Whyte. Proceedings of the IEEE International Workshop on Intelligent Robots and Systems, p1442-1447, Osaka, Japan, November 1991.

[20] "Mobile Robot Localisation and Mapping in Extensive Outdoor Environments" by I. Bailey. PhD thesis, ACFR, University of Sydney, Australia, August 2002. 